Journal of The Magnetics Society of Japan Vol. 13, Supplement, No. S1 (1989)

(C) 1989 by The Magnetics Society of Japan

\title{
ION BEAM DEPOSITION OF CO-Cr FILMS BY USING OPPOSED TARGETS SPUTTERING TECHNIQUE
}

\author{
Yoichi HOSHI* and Masahiko NAOE** \\ * Tokyo Institute of Polytechnics, Atsugi-shi, Kanagawa-ken 243-02 Japan \\ Tokyo Institute of Technology, Meguro-ku, Tokyo 152 Japan
}

\begin{abstract}
In order to deposit Co-Cr film with excellent c-axis orientation, ion beam deposition of the $\mathrm{Co}-\mathrm{Cr}$ films has been attempted by using an opposed targets sputtering apparatus, since it can be operated at a gas pressure as low as $1 \times 10^{-4}$ Torr and many metal ions are produced in the plasma during sputtering. The ions are extracted from the plasma through a grid and are deposited directly on a substrate. However, the ions in the plasma cannot reach to the substrate if the substrate is at earth potential, since the plasma is at a negative potential. Deposition rate $\mathrm{Rd}$ increases gradually as substrate potential Vsub decreases from $\mathrm{OV}$ and takes a maximum value of about $8 \AA / m i n$ at a Vsub around $-60 \mathrm{~V}$. Rd decreases steeply as further decrease in Vsub from -70 to $-90 \mathrm{~V}$. Crystal structure and magnetic properties of the film are also changed with Vsub, though c-axis orientation is not observed.
\end{abstract}

\section{INTRODUCTION}

Ion beam deposition is one of the most attractive film deposition technique because of its high potential of low deposition temperature in the formation of the film with good crystallographic structure [1-4]. In opposed targets sputtering, high rate sputtering can be performed at a gas pressure as low as $1 \times 10^{-4}$ Torr [5] and many metal ions are produced in the plasma during the high rate sputtering[6]. Therefore, if an ion extraction grid is arranged in the plasma and is applied a negative potential compared to the plasma, the metal ions in the plasma can be extracted through the grid and can be deposited directly on a substrate. Thus, it seems that ion beam deposition can be easily performed by utilizing the opposed targets sputtering apparatus, since the mean free path of the ions at a Ar gas pressure of $1 \times 10^{-4}$ Torr is larger than 50 $\mathrm{cm}$.

Co-Cr film should have excellent caxis orientation to use for perpendicular magnetic recording media. So, the ion beam deposition method is very attractive to realize the Co-Cr film with excellent c- axis orientation. In this work, we tried to deposit the Co-Cr film by the new ion beam deposition technique where an opposed targets sputtering technique was applied. In this paper, we will show the operating characteristics of the ion beam deposition system and the structure and magnetic properties of the Co-Cr film deposited by the method.

\section{ION BEAM DEPOSITION SYSTEM}

Figure 1 shows a schematic diagram of the opposed targets sputtering apparatus used in this work. Co-Cr alloy (21 at.\%(r-Co) plates of $13 \times 18 \times 0.5 \mathrm{~cm}$ in size were used as the targets. An ion extraction grid and a substrate were arranged as shown in the figure so that the ions in the plasma are extracted through the grid and are deposited directly on the substrate. The substrate is located at the position where the most of neutral sputtered particles emitted from the targets cannot be arrived directly.

Figure 2 shows typical discharge characteristics of this sputtering apparatus at various argon gas pressure. It is evident from the figure that the sputtering can be performed at a very low gas pressure as low as $0.15 \mathrm{mTorr}$ where the 


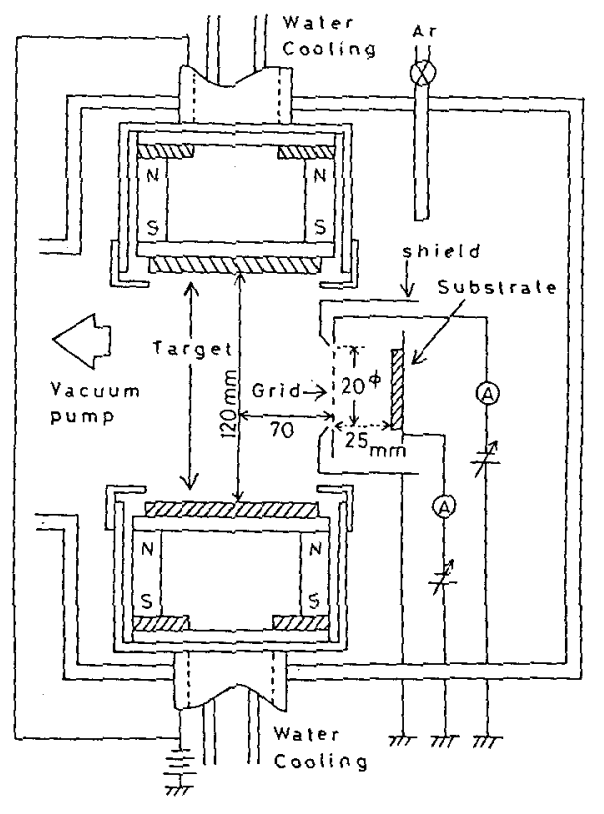

Fig.1 A schematic diagram of the ion beam deposition system utilizing an opposed targets sputtering system.

mean free path of the sputtered particles is much larger than the distance between the substrate and grid, though the applied voltage to the targets should be increased as the gas pressure decreased.

Figure 3 shows the changes in plasma potential with the position in the plasma at the various sputtering gas pressures. These plasma characteristics was measured with a conventional Langmuir probe method. The plasma potential $\mathrm{Vp}(\mathrm{V})$ takes a negative value and increases as the sputtering gas pressure increases. Therefore, if the substrate is at a potential of $V s u b(V)$, the ion, incident to the substrate, is thought to have an energy about e(Vp-Vsub) (eV). Thus, the ions ir plasma cannot reach the substrate if the substrate is at earth potential.

Figure 4 shows the energy distribution of ions incident to the substrate. A schematic diagram of the measuring system for the energy distribution is shown

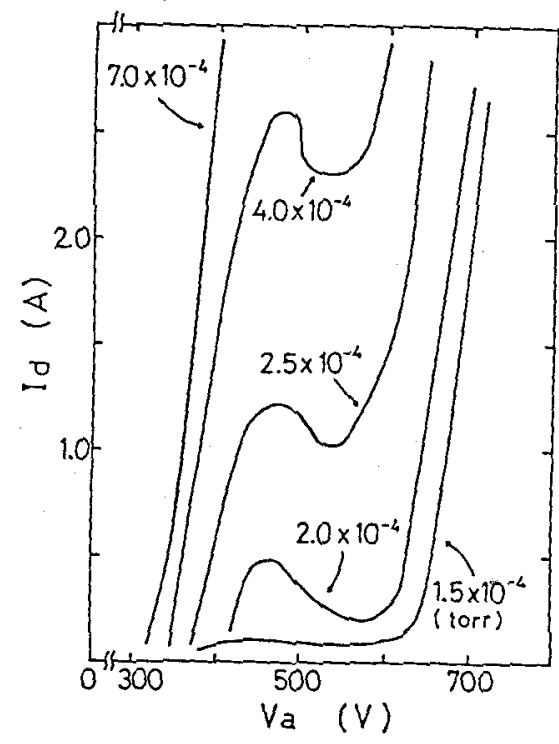

Fig.2 Typical discharge characteristics of the opposed targets sputtering system at various gas pressures.

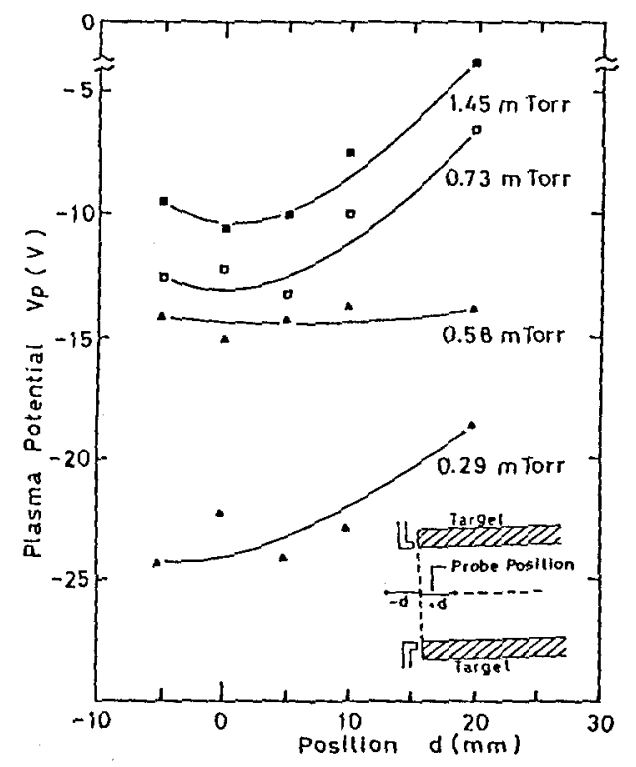

Fig. 3 Changes in plasma potential Vp with the posjtion in the space between the targets at various sputtering gas pressure. 


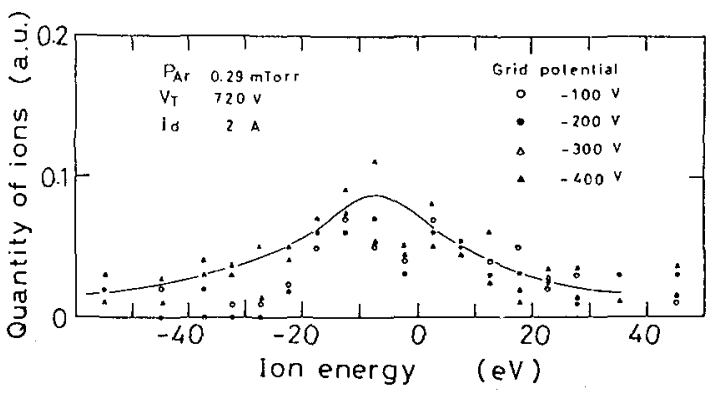

Fig.4 Energy distributions of ions incident to the substrate.

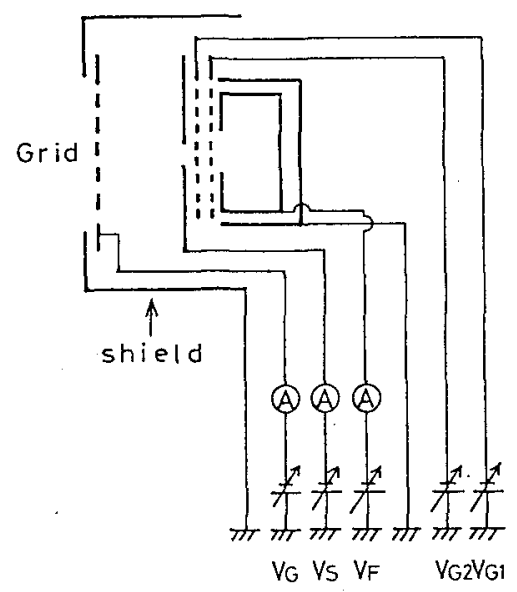

Fig.5 A schematic diagram of the measuring system of the ion energy distribution.

in Fig.5. It is evident from the figure that the ions have an energy about $-10 \mathrm{eV}$ and the energy distribution depends little on the grid potential. This result i.e., the ion has negative energy, is caused by the fact that the plasma potential has a negative value as shown in Fig.3. However, it should be noted that Ar ions are also extracted together with metal ions in this deposition technique.

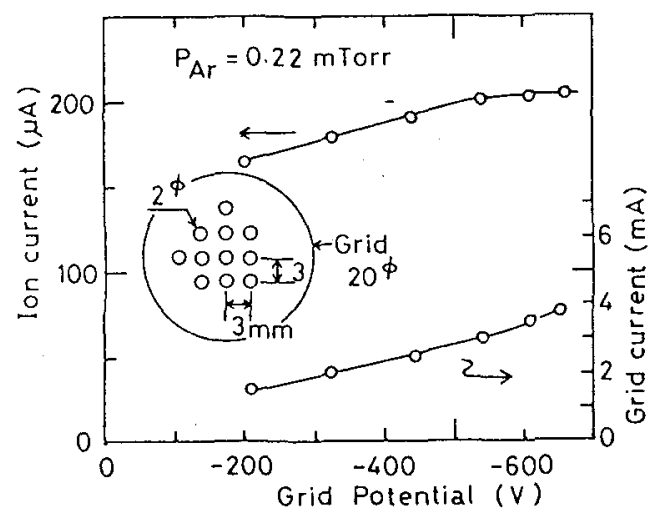

Fig. 6 Changes in the ion current at substrate holder and the grid current with ion extraction grid voltage.

Figure 6 shows the changes in the ion current at substrate holder and grid current with applied grid voltage. The ion current increases gradually with an increase of grid voltage, though the ion current is less than $1 / 10$ of the grid current. The ion current may be increased by enlarging the grid hole area.

\section{FILM PREPARATION CONDITIONS AND MEASUREMENT}

The films about $1000 \AA$ thick were deposited on a silicon wafer substrate at the argon gas pressure of about 0.3 mTorr. In order to clarify the effect of the energy of the incident ions on the structure of the film, films were deposited at various substrate potential. Typical film preparation conditions are listed in Table 1. Figure 7 shows the changes in film deposition rate $R d$ with substrate bias voltage Vsub. Rd increases gradually as substrate potential Vsub decreases from 0 $\mathrm{V}$ and takes a maximum value of about 8 $\AA /$ min at a Vsub around $-60 \mathrm{~V}$. Rd decreases steeply as further decrease in Vsub from -70 to $-90 \mathrm{~V}$. The increase in the $\mathrm{Rd}$ as Vsub decreases from 0 to $-30 \mathrm{~V}$ may be caused by the increase of arriving metal ions to the substrate. While, the decrease 
Table I Film deposition conditions.

\begin{tabular}{|c|c|c|}
\hline Sputtering gas pressure & 0.3 & mTorr \\
\hline Target voltage & 600 & $\mathrm{~V}$ \\
\hline Discharge current & 2 & A \\
\hline \multicolumn{3}{|l|}{ Ion extraction } \\
\hline grid voltage & -400 & $\mathrm{~V}$ \\
\hline Substrate bias voltage & $+30-90$ & V \\
\hline Substrate & Silicon & wafer \\
\hline
\end{tabular}

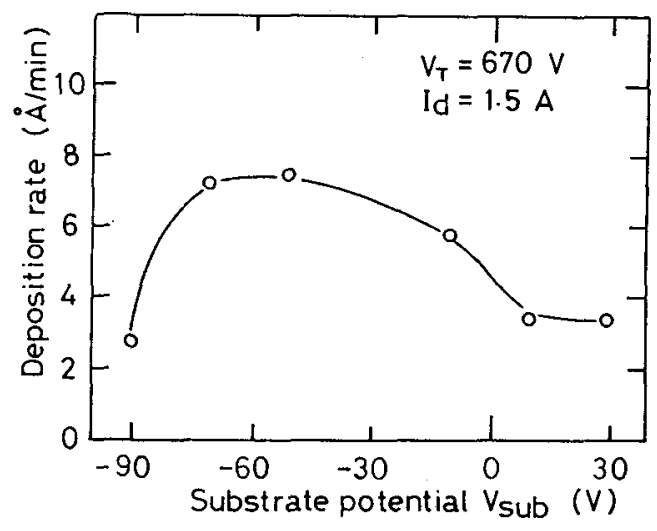

Fig.7 Changes in film deposition rate $\mathrm{Rd}$ with substrate bias voltage Vsub.

in Rd as Vsub decreases from $-60 \mathrm{~V}$ to -90 $\mathrm{V}$ should be attributable to the decrease in sticking probability of the arriving ions or an increase in resputtering because of the increase of the kinetic energy of the incident ions. The fact that films are deposited on the substrate even if the substrate potential is at a Vsub of $30 \mathrm{~V}$ suggests that some neutral atoms are arrived at the substrate.

Crystal structure and c-axis orientation was characterized with X-ray diffractometry. The coercive force Hc and saturation magnetization $M s$ of the film were measured with a VSM.

\section{STRUCTURE AND MAGNETIC PROPERTIES OF THE FILM}

Figure 8 shows the changes in $X$ ray diffraction diagrams of the film with substrate bias voltage. It is evident from the figure that the crystallites in all of the film has random orientation, though the crystallinity changes a little with Vsub. Especially, the film deposited at a Vsub around $-50 \mathrm{~V}$ has amorphous like structure. But the reason is not clear till now.

Figure 9 shows the changes in coercive force $\mathrm{Hc}$ of the film with the substrate bias voltage. Coercive force takes a minimum value at a Vsub around $-50 \mathrm{~V}$. This can be attributable to the fact that the film deposited at the Vsub has inferior crystallinity. Saturation magnetization of these film is in the range from 300 to $400 \mathrm{emu} / \mathrm{cc}$, though its dependence on the Vsub was not clear. Typical $M-H$ loops of these films are shown in Fig.10. The M-H curves indicate that these films have very little perpendicular magnetic anisotropy.

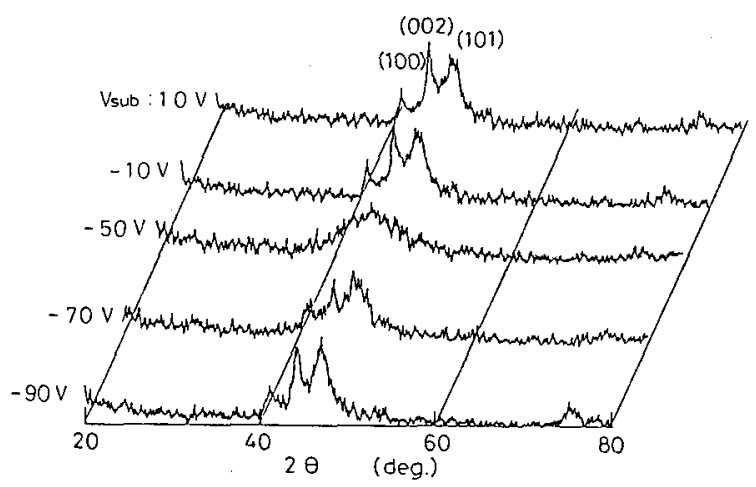

Fig. 8 Changes in $X$ ray diffraction diagrams of the film with substrate bias voltage. 


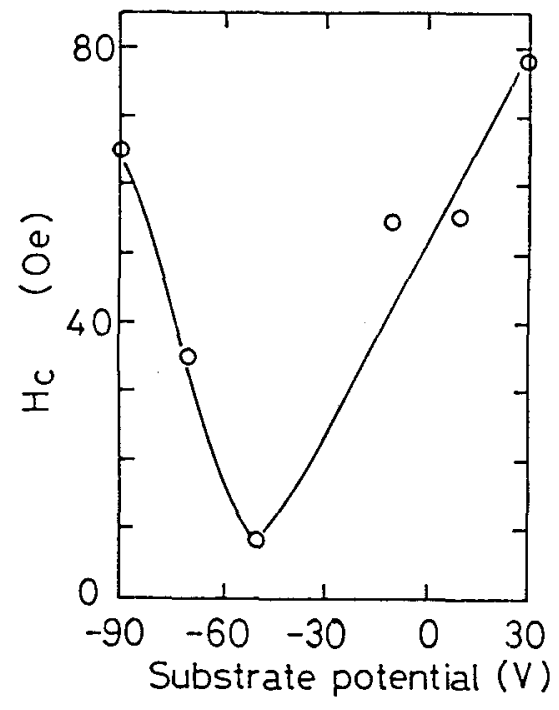

Fig.9 Changes in coercive force $\mathrm{Hc}$ of the film with substrate bias voltage Vsub.

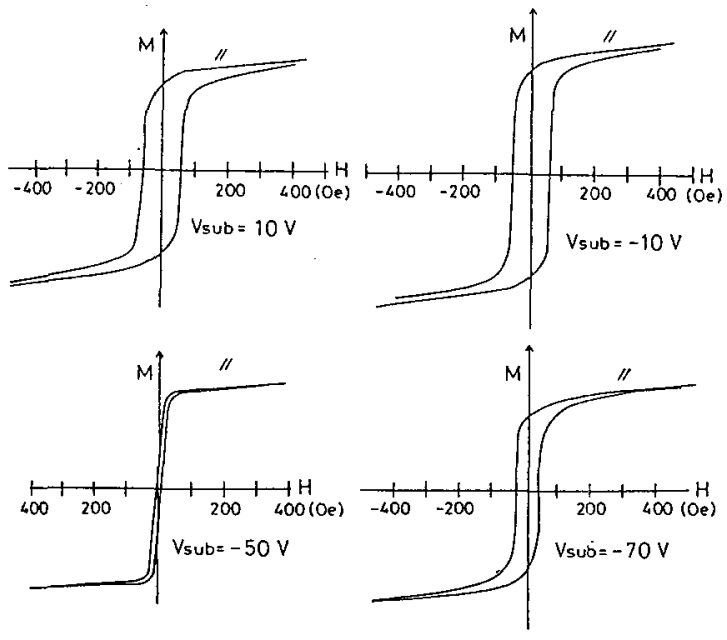

Fig.10 Changes in M-H curves of the film with substrate bias voltage.

\section{CONCLUSION}

Ion beam deposition of the Co-Cr films has been attempted by using an opposed targets sputtering apparatus and following results are obtained.

(1) Ion beam deposition can be easily performed by utilizing the opposed targets puttering technique.

(2) The ions extracted from the plasma cannot reach to the substrate if the substrate is at earth potential, since the plasma is at a negative potential.

(3) Deposition rate Rd increases gradually as substrate potential Vsub decreases from $O V$ and takes a maximum value of about $8 \AA / \mathrm{min}$ at a Vsub around $-60 \mathrm{~V}$. Rd decreases steeply as further decrease in Vsub from -70 to $-90 \mathrm{~V}$.

(4) Crystal structure and magnetic properties of the film are also changed with Vsub, though c-axis orientation is not observed.

However, we could not clarify the advantage of the ion beam deposition technique for the deposition of Co-Cr films. Therefore, further investigation must be continued.

\section{REFERENCES}

[1]J.L.Vossen and W.Kern, "Thin Film process", Academic Press,pp.198-203(1978) [2]J.Amano and R.P.W.Lawson; J. Vac. Sci. Technol., 14,831(1977) and 14,836(1977) [3]S.Aisenberg, and R.Chabot; J.Appl. Phys. , 42,2953(1971)

[4]Y.Hoshi,M.Naoe and S.Yamanaka; Trans. IECE Japan, J65-C,490(1982) (in Japanese)

[5] N.Terada, Y.Hoshi, M.Naoe, and S.Yamanaka; Proc. Int'l Ion Engineering Congress-ISIAT'83 \& IPAT'83, Kyoto (1983)

[6] N.Terada, Y.Hoshi, M.Naoe, and S.Yamanaka; IEEE Trans. Mag., MAG-20,1451 (1984) 